\title{
DIALOGUE FOR DESIGN TEAMS: A CASE STUDY OF GENERATIVE CONVERSATIONS FOR DEALING WITH DIVERSITY
}

\author{
Stefan PERSAUD and Shreyas PRAKASH and Bas FLIPSEN \\ Delft University of Technology, Industrial Design Engineering, Delft
}

\author{
Keywords: Team performance, diversity, design teams, cooperation, dialogue
}

\begin{abstract}
In the Integrated Product Design master's at Industrial Design Engineering, there is a growing diversity in students. In recent years we increasingly noticed that diversity could lead to complications within the various student teams. Examples are miscommunication, frustration, and interpersonal conflicts. This growing diversity between the team members strongly appeals to their ability to deal with these complications in a constructive way. Via a KPI tracking system we kept track of team performance and we identified four aspects affecting the team dynamics within the Advanced Embodiment Design project team [1]. This paper presents the case study of one of the student design-teams which was highly dysfunctional in design approach and conflicting cultural differences. It made this group perform at a lower level. Theory $U$ [2] and dialogue techniques [3] were an unfamiliar approach for student design teams. Interventions using generative conversations stimulated connectedness between the team members and improved the team dynamics. The use of culture mapping [10] proofed to be a valuable tool to bridge the culture gap. Although this case study only describes one student design team, the drastic improvements resulted in the team winning the iF Design Talent Award 2020. The findings are promising, and further research is needed to investigate the generalizability of these approaches for Industrial Design Engineering education.
\end{abstract}

\section{INTRODUCTION}

In the master's course Advanced Embodiment Design (AED), students cooperate in design teams on a client-based design project for one semester consisting of 21 European Credits. These student design teams consist of highly diverse student members, with differences in nationality, prior education, and socio-emotional development. Each team is supervised by a teacher on a weekly basis. In previous research [1] we noticed that the diversity between the team members can lead to complications within the team. Miscommunication, frustration, and sometimes interpersonal conflicts are examples of elements that we found that lead to lower performance due to the inability of the team and the coach to dealing with these complications constructively. The design of a KPI tracking system [1] was used to monitor the student-teams' performance, which was discussed during the weekly coach meetings. We identified four aspects on the team dynamics within the AED project team affecting student-groups becoming highly functioning teams: (i) cultural differences, (ii) differences in design approach, (iii) emotional differences and (iv) differences in competencies [1]. In this case study paper, we focus on one team that addressed two out of the four aspects that frustrated the team dynamics and flow of the project: cultural differences and design approach. Within the IPD masters' programme, there has not been any previous research addressing these diversity aspects which affect the dynamics in student design teams. Students and coaches within the IDE faculty at TU Delft do not have an appropriate approach to deal and tackle these problems. The goal of this research is to describe our approach to increase the ability of the tam and their coaches to deal with these differences based on Theory $\mathrm{U}$ approach [2] and Dialogue techniques [3]. Dialogue techniques supported the student team shifting their conversations from politeness and/or debate to reflective inquiry-conversations which led to more generative conversations. Generative dialogues are conversations where the students listen not only from within themselves or from within others but from the whole system. Moving conversation to generating new understandings and knowledge rather than simply negotiating from current understandings and 
positions. In this conversational process of dialogue, the coach helped the student team to move through the Theory U process. Shifting from habits of judgement to seeing each other with a more creative open mind. Finally, the team further progressed to sensing each other with more empathy and compassion which resulted in a healthier collaborative team, incorporating their differences as an opportunity for generating design solutions. This paper presents the case study of this student design team. We describe the theory underlying the interventions of the team dynamics expert, the composition of the group, their different design approaches, and the interventions that lead to drastic improvement and resulted in the team winning the iF Design Talent Award 2020. Although this study shows promising results, further research is needed to investigate the generalizability of the findings.

\section{THEORY U, DIALOGICAL CONVERSATIONS AND CULTURE MAP}

AED student design teams are multi-diverse. They are developmental, dynamic and the social processes and context of the teams often change. This is different from the context where Tuckman [4] and Smulders [5] describe teamwork. The nature of the Tuckman model (Forming, Storming, Norming, Performing and Adjourning) is hierarchical where the following step can only be reached when the previous is accomplished. Although Smulders addresses student teams he is not explicit on diversity and the iterative developmental nature of student design teams. Miller [6] on the other hand states that groups can be viewed as systems. Personal and group related processes of creativity, introspection and intuition are more prominent today [7] where many of the design challenges far exceed the knowledge and skill that any one person might possess. This context is the reality of the AED project and therefor important to understand and improve teamwork and collaboration. Hays [9] addresses how and why Theory U applies to teams: "It is a model that transfers easily from an individual to a collective context and holds great potential to spark the learning that is necessary to improve team functioning and enable greater performance".
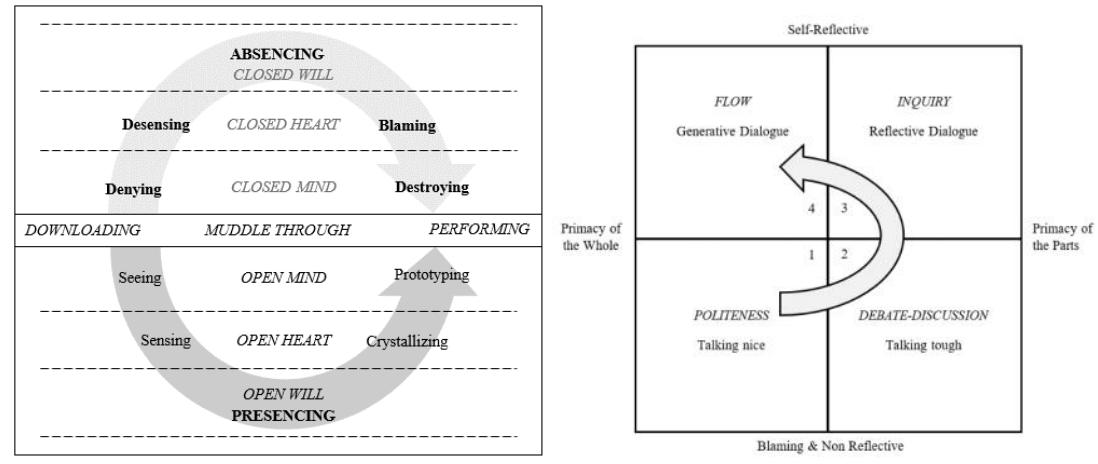

Figure 1. Theory $U$ (left) and Four types of conversations (right), adapted from Scharmer [2]

Theory $\mathrm{U}$ [2] describes two opposing processes (figure 1, left): presencing and absencing. Presencing is the process where teams collaborate and embrace emerging possibilities. Absencing is the process where teams disconnect and get stuck in old habits. In each process, three elements of communication are relevant and can be either open or closed. Communication from the 'mind' is described as (non) judgmental communication of team members based on prior experiences. Communication from the 'heart' is described as the emotional (dis) connection of team members. Communication from the 'will' is described as the ability of a team to act in an instant. Scharmer also identifies a process of muddling through, where teams are in between presencing and absencing. Before teams either go into the absencing or presencing journey, they start with a phase called downloading. This is when the team is starting to get together and engage based on past patterns.

Communication within the team is central in the team process: talking and listening. Scharmer [2] describes four types of conversations (figure 1, right). (i) Talking nice, where people listen to confirm the positive aspects, the goodwill, filtering, politeness, self-censoring. (ii) Talking tough, where people listen to respond, to counter others' arguments, such as debate, discussion, and conflict. (iii) Reflective dialogue, where people listen to understand, accepting the others perspective without feeling the need to disagree with them and allow diversity of perspectives. (iv) Generative dialogue, where people listen to the human experience of the other and what resonates with their own humanity, sensing the wholeness of which we are part, unity in diversity. Talking nice and talking tough is prominent in the absencing process and reflective dialogue and generative dialogue are applied in the presencing process. 


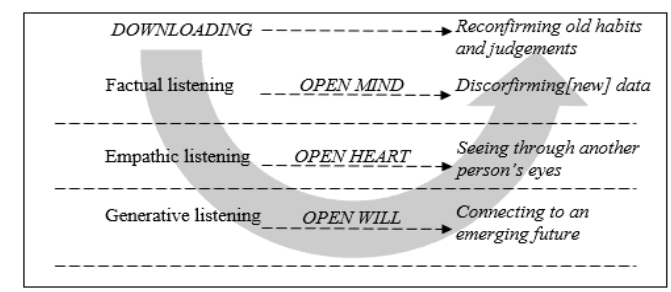

Figure 2. Four levels of listening adapted from Scharmer [2]

Within the fields of conversation, Scharmer [2] also describes four levels of listening that support the process within teams for transferring "Reflective Dialogue" to "Generative Dialogue" (figure 2): (i) Downloading: listening from habits of judgement, (ii) Factual listening: listening from outside and noticing differences, listening with an open mind,' (iii) Empathic listening: listening from within, sensing, listening with an 'open heart' and (iv) Generative listening: listening from source, from what is emerging, listening with an 'open will'.

Table 1. Eight Dimensions of The Culture Map [10].

\begin{tabular}{|l|c|r|}
\hline \multicolumn{3}{|c|}{ Eight dimensions of the Culture Map } \\
\hline Extreme A & Key area & Extreme B \\
\hline Low-context & Communicating & High-context \\
\hline Direct negative feedback & Evaluating & Indirect negative feedback \\
\hline Egalitarian & Leading & Hierarchical \\
\hline Consensual & Deciding & Top-down \\
\hline Task-based & Trusting & Relationship-based \\
\hline Confrontational & Disagreeing & Avoids confrontation \\
\hline Linear-time & Scheduling & Flexible-time \\
\hline Principles-first & Persuading & Application first \\
\hline
\end{tabular}

Besides group dynamics, cultural differences have a large effect on the team's performance. The Culture Map [10] is a model that defines eight areas where cultures vary along a spectrum between two opposing extremes (Table 1). It provides a framework for teams that face cultural differences. Team members analyse the position of their culture relative to one another. This enables them to decode how culture influences their collaboration. The space in between the extremes can be considered as a continuum. Within the range of behaviours of a given culture, individual differences occur. The goal of using the model is to support interacting between team members and improve watching more, listening more, and speaking less.

\section{CASE STUDY EQUARUN}

The AED project 'Equarun' is concerned with developing an assistive device to help people with deaf blindness. This device must be capable of transmitting complex instructions on road conditions, striking a good balance between freedom and safety. The team consisted of three Chinese design engineering students, a Spanish student from mechanical engineering and an Indian student educated as an electrical engineer. Both the company coach and the AED coach are Dutch. The company coach has a master's in medical research and the AED coach has a master's in Industrial Design Engineering achieved at the Delft University of Technology. Although most students in the team have different competencies, it did not play a role in team-dynamics and the team did not encounter any emotional differences. The two emerging difficulties in this group were based on differences in design approach and nationality.

A few weeks into the project, different design approaches were leading to a certain amount of incongruity. A part of the student team approached design as a process where designers decide which design is meaningful for the user. Another part of the team approached design as a process where user input should be steering the design process. Indirectly the company insisted the team should conduct more extensive tests with the deafblind runners to support all conclusions. The confusion in these approaches led to discussions and friction in communication. It also led to differences in the way the team planned the project. The cultural differences were compounding the communication gap. Certain information was received amongst the team members in a roundabout way with insufficient direct communication. The team members found it difficult to express pressing pain-points openly as the assumption was that it might offend someone. They realized it was also noticeable in nonverbal communication of their respective team members. The bonding glue to express openly in a safe space was missing. Halfway through the project, the mid-term assessment was approaching. The team assigned tasks (both as an individual and as a group) but they did not deliver on time. The team was struggling in managing the different demands of several stakeholders. The coach from the Running 
Blind Foundation was giving directions which were not in sync with the academic requirements for the course.

\section{APPROACH}

To keep track of the team development, the coach scored the student team on 3 strategic and 4 operational Key Performance Indicators weekly [1]. Each KPI ranges from 1 (bad) up to 5 (good). The score is accompanied by textual journaling from the coach about the design team's performance. Based on these scores the coaches discuss the most dysfunctional teams on a weekly basis. The case study team discussed in this paper was pinpointed as dysfunctional in an early phase of the course. In this paper we compared the coach's journal next to that of the student team journal, which was developed in hindsight. Based on those discussions and referring to Theory $\mathrm{U}$ processes [2], it is decided what kind of interventions could improve the team dynamics of the student team. In this particular team, we used two experts to address the cultural differences within the team and an expert in the field of dialogue to have generative conversations with and within the team. The expert on cultural differences consulted the student team and provided the Culture Map [10] as a means to better understand each other. The team discussed the dimensions of the model and they constructed their own map using standard scores for Dutch, Chinese, Spanish and Indian culture (figure 3). The expert on dialogue mediated several meetings with the group and some sessions with individual team members. In these sessions, the expert explained Theory $\mathrm{U}$, the dialogue principles and demonstrated the practice of dialogue [3]. When the interventions were successful it could be noticed in higher scoring on the KPI by the coach. To see if there is a difference in the experience of the interventions this specific team also recorded KPI's in hindsight as a reflection exercise after finishing the project.

\section{RESULTS}

\subsection{The Culture map and KPI scores}

When mapping the team's cultural backgrounds onto the Culture Map framework [10], certain points of tension became evident (figure 3). Most prominent difference is the persuasion parameter. It explains the confusion in the approach of the design process. Another parameter that caused tension corresponds to the deciding parameter. As the student team cultures were fundamentally more top-down, the project manager played a key role in steering the team towards the key milestones. Apart from this, the cultural differences between the team, coach and the client created difficulties in the parameter of 'communication' as the team was quite used to high-context communication and indirect negative feedback.

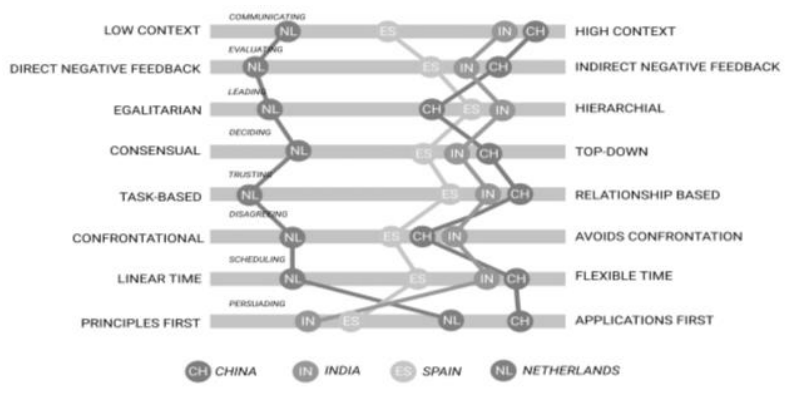

Figure 3. Team scan on The Culture Map [10]

Each week the coach scored the team on 7 KPI's [1]. The student team wrote their KPI journal in hindsight after consulting the whole team using recorded notes from team meetings and both individual and team reflections. These reflections were compulsory exercises of the course [11]. We briefly discuss how the scoring on the four operational indicators, (i) project management approach, (ii) planning, (iii) group dynamics and (iv) perceived stress level, developed during the project. In the first ten weeks the coach scored the Project Management low. The design progress was disturbed due to the lack of wellprepared coach meetings and poor communication with the coach. From week 10 on the team became more proactive and kept the coach up-to-date. In week 8, Group dynamics had a sharp downward move but improved following regular constructive feedback sessions, supportive reflective dialogue, and improved team bonding. As the team enjoyed the work in after the midterm (week 10), the perception 
of stress was lower and much more manageable. The first 10 weeks the team was failing to match their target weekly goals and the KPI scores on 'Project Management' were low. After guided interventions on dialogue and Culture Map the team communication improved and helped them achieve the goals better. The completion of tasks, scored in 'Planning on Time completion' went down in week 16-17, due to the confusing mismatch in the expectations between the coach and client. The client was expecting research outcomes to be more extensive which were not manageable within the course outcomes. Higher scores on Group Dynamics were due to the expert supporting the team shifting from talking politely and discussions to factual, empathic, and generative listening. A peak stress level occurred during the mid-term in week 10 . The team felt the risk of failing to match the expectations of the course. The stress gradually went down because of the reflective dialogues, which helped them become more self-aware. Finally, the team started communicating with the coach and the client more proactively.

\subsection{Theory U - Open Mind, Open Heart, Open Will}

After a series of events during project week 8-10, the company coach informed the course coordinators that the progress made by the team was not satisfactory. This led to the coach informing the team they might fail the course. The coach advised to restructure the design process to bring more clarity. The dialogue coach facilitated guided dialogue sessions with the AED coach and the team. In these sessions, the dialogue coach provided theory, instructions and moderated the conversations building the skills of listening and talking. To improve each of the team members individually, he also arranged 1:1 conversation. During these dialogues, questions were asked about the team's approach to design, analysis of what went wrong, and what could be the steps which we could take. The team was given two weeks to improve, and they bonded over the crisis. The two-week notice helped them formulate their shortcomings and reflect on a better way forward, shifting from absencing to presencing [2]. As actionable steps forward, they started sharing daily updates proactively with the client, asking for the client's feedback on the design tests which they would be conducting with the deafblind and the fact that they could not conduct design tests with real deaf-blind runner groups. They planned a test within a short timeline so they could present their progress for the follow-up meeting with AED coach and dialogue coach. In the follow-up meeting, both coaches felt that the design process and planning was much more structured and coherent. They gave the team 'the green signal', which in a way meant that the process the team had revamped was in the right direction. The restructuring helped them to connect to each other and understand their limitations.

During week 11, the team started interacting more with the deafblind runners after the first major test. They started getting more closer to them, connecting, and understanding them better. This was the onset of feeling 'empathy' for the user. To put their feet in their shoes and literally 'running' with it, they volunteered as runners for the Running Blind Foundation to guide the deafblind runners. This helped them to also understand and feel the pain points of the guides who were paired with these runners.

At this moment during week 12-13, the team felt that they were getting united by their vision - to impact the lives of the deafblind runners. When the team started meeting the runners and seeing them up close, the feeling of empathy started taking shape. It was a turning point for them as a team as the initial inhibitions and the barriers were slowly dissolving. The team connected more socially with the community of runners: they participated in the Rotterdam Marathon in association with the Running Blind Foundation and participated in the Running Blind Week as runners. All these activities helped them to integrate with the runner's community, and there was a heightened sense of belonging.

Owing to all this, the team made it a point to schedule at least one design test with the runners every week during week 14-20. The design tests were validated by the client and the coach and were conducted at the AV40 running track at Delft. These tests were conducted for design iterations. The team also conducted extensive tests with deaf-blind runners from Leiden, den Bosch and Rotterdam, and they noticed they could keep repeating the same iterative design process every week. After the completion of the embodiment of the final product, it was tested with 10 deafblind runners and their guides from various locations in the Netherlands. Although the client wanted more tests, they had to stop at a point as they also had their course requirements for completing in time. The team was not afraid to communicate these issues with their coaches. After the presentation, the team still felt a sense of inadequacy as designers as the mid-term presentation was a profound moment in this project. After a year and seeing all the deafblind runners giving strong feedback on our product and even the 
organization, Running Blind Foundation wanted to take the product forward with their manufacturing partner, R2PRO. The team was encouraged by this and applied for the iF Design Talent Award 2020.

\section{CONCLUSION AND DISCUSSION}

In this paper we have discussed the case study of one AED student team on team dynamics. Out of our previous research [1] we identified the key issues concerning four types of diversity (cultural, design approach, emotional and competencies). In this paper we have researched the student team and the coach perspective on issues concerning differences in cultures and design approach when working in a design team. By weekly tracking seven KPI's and the accompanying qualitative journaling from students and coach, we could identify the moments and issues when team dynamics was deteriorating or improving. Although the student team generated the KPI scores and team journal in hindsight, the figures follow a similar path. Timely interventions by experts were taken to create awareness and conversational skills were used to improve the team dynamics. The Theory U [2] seems to provide a good framework for monitoring the aspects in the process. It also provides a pathway via open mind, open heart to presencing where this group of students could become a more effective design team. The focus on communication with the dialogue approach of talking and listening [3] seems to be a key element in improving the collaboration within the team and with the coaches. The culture map [10] is a valuable tool to raise awareness of cultural differences. Teams can now visualize where they differ and find improvements. With the use of dialogue, the team can shift from debate to reflection and generative conversations. After the expert mediated dialogue sessions, the KPI scores went up and without the expert the KPI scores went down again. This could indicate that the team and coach were able to cooperate when facilitated initially and in the learning process of understanding and applying dialogue themselves. The KPI scores from the student perspective in final last weeks were high. This indicated that the team was collaborating very well with hardly any discussions. The generative dialogue conversations had become a habit in the designing process. Some questions remain about the case we presented. We only described one case and we need further research to see if these findings are generalizable for other teams within the course. Should we inform all teams and coaches, for instance with a briefing or lecture about the Culture Map? Not all teams are subjected to cultural differences when cultures are close in most dimensions. The student team KPI's generated in hindsight makes the reliability of the data debatable. We therefore recommend letting student teams keep track of their own weekly KPI. Could KPI tracking by all student teams also support their self-reflection? More generally we are wondering if other design teams and coaches would also improve their team dynamics using dialogue as communication tool to overcome talking nice and talking tough in the design process? Could it be that Theory $\mathrm{U}$ and dialogue are overlooked perspectives in design education at the faculty of IDE at TUDelft? This research has received funding from the Comenius Teaching Fellowship funded by the NRO in the Netherlands.

\section{REFERENCES}

[1] Flipsen B. and Persaud S. M. Handle with care: coaching multi-diverse project groups to become healthy design teams, E\&PDE 2020, University college, Herning, Denmark, 2020.

[2] Scharmer O. Theory U, leading from the Future as it Emerges, Berett-Koehler Publishers, 2016.

[3] Isaacs W. Dialogue and the art of thinking together, Currency, 1999.

[4] Tuckman B. W. and Jensen M. A. C. Stages of small-group development revisited. Group Organization Management, 2(4), 419-427, 1977.

[5] Smulders F., Brehmer M., and Meer van der H. TeamWorks, by students, for students. Mozaic Business Publishers, Delft, 2012.

[6] Miller D. L. The Stages of Group Development: A Retrospective Study of Dynamic Team Processes. Canadian Journal of Administrative Science, 20(2) pp. 121-134, 2003.

[7] Senge P., Scharmer O., Jaworski J., and Flowers B. S. Presence: Human purpose in the field of the future. Cambridge, MA: The Society for Organizational Learning, 2004.

[8] Flipsen B., Persaud S. M., and Magyari R. Student's perspectives on challenges within multidiverse design teams. E\&PDE 2020, University college, Herning, Denmark, 2021.

[9] Hays J. Theory $U$ and team performance: Presence, participation, and productivity, Perspectives on Theory U: Insights from the Field. Chapter: 10, IGI Global, 2016.

[10] Meyer E. The Culture Map, Public Affairs, New York, 2014.

[11] Gordijn F. et al. Reflection Methods, Wageningen Centre for Development Innovation, Wageningen University \& Research, 2018. 